\title{
DISEÑO Y DESARROLLO DE UNA APLICACIÓN PARA DISPOSITIVOS MÓVILES PARA EL SEGUIMIENTO Y CONTROL DE LA ENFERMEDAD DE MÉNIĖRE
}

\section{Design and development of a mobile app for Ménière disease patients}

\author{
Jorge REY-MARTÍNEZ1; Juan Manuel ESPINOSA-SÁNCHEZ² \\ ${ }^{1}$ Departamento de Otorrinolaringología. Hospital Universitario Donostia. San Sebastián. España. \\ 2 Servicio de Otorrinolaringología. Hospital Universitario Virgen de las Nieves. Granada. España. \\ Correspondencia: bendermh@hotmail.com
}

Fecha de recepción: 16 de abril de 2018

Fecha de aceptación: 21 de abril de 2018

Fecha de publicación: 23 de abril de 2018

Fecha de publicación del fascículo: 15 de marzo de 2019

Conflicto de intereses: Los autores declaran no tener conflictos de intereses

Imágenes: Los autores declaran haber obtenido las imágenes con el permiso de los pacientes

Política de derechos y autoarchivo: se permite el autoarchivo de la versión post-print (SHERPA/RoMEO)

Licencia CC BY-NC-ND. Licencia Creative Commons Atribución-NoComercial-SinDerivar 4.0 Internacional

Universidad de Salamanca. Su comercialización está sujeta al permiso del editor

RESUMEN: Introducción y objetivo: La aplicación de las tecnologías de la información y la comunicación al cuidado de la salud está contribuyendo a una verdadera revolución en la asistencia sanitaria. La medicina actual se está beneficiando del rápido y extraordinario desarrollo tecnológico de los teléfonos móviles «inteligentes» y tabletas. La medicina móvil ( $m$ Health) permite, entre otras ventajas, una mejor monitorización de la sintomatología de los pacientes. Esto no solo facilita su seguimiento clínico, mejorando la atención, sino que contribuye a que los pacientes sean corresponsables de su propia salud, todo lo cual ha de contribuir a mejorar su calidad de vida. Las aplicaciones informáticas diseñadas específicamente para estos dispositivos $(a p p)$ son especialmente útiles para monitorizar enfermedades crónicas, como lo es la enfermedad de Ménière. Método: En este trabajo se expone el proceso de diseño, desarrollo y publicación de una aplicación denominada «Ménière» destinada a ayudar a los pacientes y sus médicos al control y seguimiento diario de esta enfermedad. Resultados: La aplicación se desarrolló para sistema operativo iOS empleando el entorno de desarrollo integrado Xcode 8.0 y utilizando Swift y Objective-C como lenguajes 


\section{DISEÑO Y DESARROLLO DE UNA APLICACIÓN PARA DISPOSITIVOS MÓVILES PARA EL SEGUIMIENTO Y CONTROL DE LA ENFERMEDAD DE MÉNIÈRE REY-MARTÍNEZ J Y ESPINOSA-SÁNCHEZ JM}

de programación. Se prestó especial atención a la privacidad, seguridad, usabilidad, veracidad y transparencia. Conclusiones: Esta aplicación proporciona al paciente una herramienta completa y versátil para monitorizar sus síntomas.

PALABRAS CLAVE: Enfermedad de Ménière; aplicación para dispositivo móvil; app; software, mHealth; audición; acúfeno.

SUMMARY: Introduction and objective: The application of information and communication technologies to health care is contributing to a real revolution in health care field. Current medicine is benefiting from the rapid and extraordinary technological development of "smart" mobile phones and tablets. The mobile medicine (mHealth) allows, among other advantages, a better monitoring and tracking of the symptomatology of the patients. This fact not only facilitates the clinical follow-up, improving care, but also helps patients to be jointly responsible for their own health, contributing to improve their quality of life. Computer applications designed specifically for these devices (apps) are especially useful for monitoring chronic diseases, such as Ménière's disease. Method: In this work, the process of design, development and publication of an app called "Ménière" is detailed, this app is designed to help patients and their doctors to track and follow this disease. Results: The app was developed for the iOS operating system using the Xcode software development kit, using Swift and Objective-C as programming languages. Special attention was paid to privacy, security, usability, veracity and transparency. Conclusions: This application provides the patient with a complete and versatile tool to monitor their symptoms.

KEYWORDS: Ménière disease; hydrops; app; software; mHealth; hearing; tinnitus.

\section{INTRODUCCIÓN}

Desde su introducción por Apple en 2007, los teléfonos móviles «inteligentes» en su diseño actual han pasado a formar parte del día a día de la mayoría de nosotros. Mucho más allá de su uso como teléfono estos dispositivos permiten a los usuarios una amplia gama de recursos de computación y comunicación hasta entonces exclusivos de los ordenadores personales. A estas características técnicas hay que añadirles lógicamente las propiamente aportadas por los teléfonos, su capacidad de movilidad y su uso intuitivo. Quizás por estos o por otros motivos hemos vivido sin duda una increíble difusión de estos dispositivos que han logrado además incorporarse o ayudarnos a realizar muchas de nuestras tareas cotidianas.

Las actividades sanitarias no se han escapado del uso de estas tecnologías para su supuesto beneficio propio y en este sentido en los últimos años ha surgido toda una corriente tecnológica que aúna sanidad y tecnología móvil, denominada «medicina móvil» (mHealth) [1,2], que en realidad es una rama de la «medicina electrónica» (eHealth).

La enfermedad de Ménière (EM) es un trastorno crónico del oído interno caracterizado por episodios recurrentes de vértigo espontáneo, de entre 20 minutos y 12 horas de duración, asociados a hipoacusia neurosensorial fluctuante, acúfenos y taponamiento ótico [3]. Los síntomas auditivos a menudo son unilaterales, aunque en un $20 \%$ a $46 \%$ la enfermedad puede afectar a ambos oídos. En un 5\% a15\% de los pacientes existe una historia familiar.

La etiología es desconocida, aunque se sospecha una base genética o autoinmune que junto con factores medioambientales condicionarían la aparición de un hydrops endolinfático, que sería el substrato anatomopatológico de la EM.

El diagnóstico de EM es clínico, conforme a los criterios establecidos por el Comité de Clasificación 


\section{DISEÑO Y DESARROLLO DE UNA APLICACIÓN PARA DISPOSITIVOS MÓVILES PARA EL SEGUIMIENTO Y CONTROL DE LA ENFERMEDAD DE MÉNIẼRE REY-MARTÍNEZ J Y ESPINOSA-SÁNCHEZ JM}

de la Bárány Society [4]. La enfermedad muestra heterogeneidad clínica, habiéndose descrito recientemente 10 subtipos o variantes clínicas de EM [5, 6], este hecho hace que la caracterización clínica de los síntomas que experimenta el paciente con EM sean la pieza clave en su diagnóstico y control.

El propósito del presente artículo es el de detallar y discutir el proceso de diseño, desarrollo y publicación de una aplicación para dispositivos móviles llamada «Ménière» para la asistencia de los pacientes con EM en el control y seguimiento diario de su enfermedad.

\section{MATERIAL Y MÉTODO}

La aplicación Ménière se desarrolló por los autores de este artículo empleando el programa de desarrollo de aplicaciones para sistema operativo iOS (Apple Inc. Cupertino, CA. EE. UU.) Xcode versión 8.0 (Apple Inc. Cupertino, CA. EE. UU.) empleando Swift y Objective-C como lenguajes de programación. El proceso de diseño y programación se realizó a lo largo de los tres últimos meses del año 2016 y el primer mes de 2017. Como motor de la base de datos de esta aplicación se empleó el sistema Core Data (Apple Inc. Cupertino, CA. EE. UU.).

\section{RESULTADOS}

\section{Diseño general de la interfaz de usuario}

El diseño de la aplicación se estableció en torno a una serie de pantallas navegables donde cada una de las funciones de la aplicación son llevadas a cabo. En la Figura 1 se muestran estas pantallas principales que se describen a continuación.

- Pantalla de bienvenida (Figura 1 A): Pantalla de inicio por defecto de la aplicación, en ella se solicita la contraseña alfanumérica o biométrica, mediante huella dactilar, para acceder a la agenda de eventos del paciente.

- Pantalla de agenda (Figura $1 \mathrm{~B}$ ): A través de ella se muestra el calendario donde aparecen marcados los días que ha habido eventos relacionados con la EM, en ella aparecen las acciones fundamentales a realizar en la agenda: consultar los eventos guardados, añadir los eventos y la creación de un informe con los eventos de cada mes. Cada una de estas acciones es controlada por su pantalla correspondiente.

- Pantalla de Información (Figura 1C): En ella se muestra una pequeña guía de información acerca de la EM, su definición actual, sus síntomas, diagnóstico, tratamiento y hábitos de vida recomendados para el paciente con EM [3-6]. 


\section{DISEÑO Y DESARROLLO DE UNA APLICACIÓN PARA DISPOSITIVOS MÓVILES PARA EL SEGUIMIENTO Y CONTROL DE LA ENFERMEDAD DE MÉNIÈRE REY-MARTÍNEZ J Y ESPINOSA-SÁNCHEZ JM}

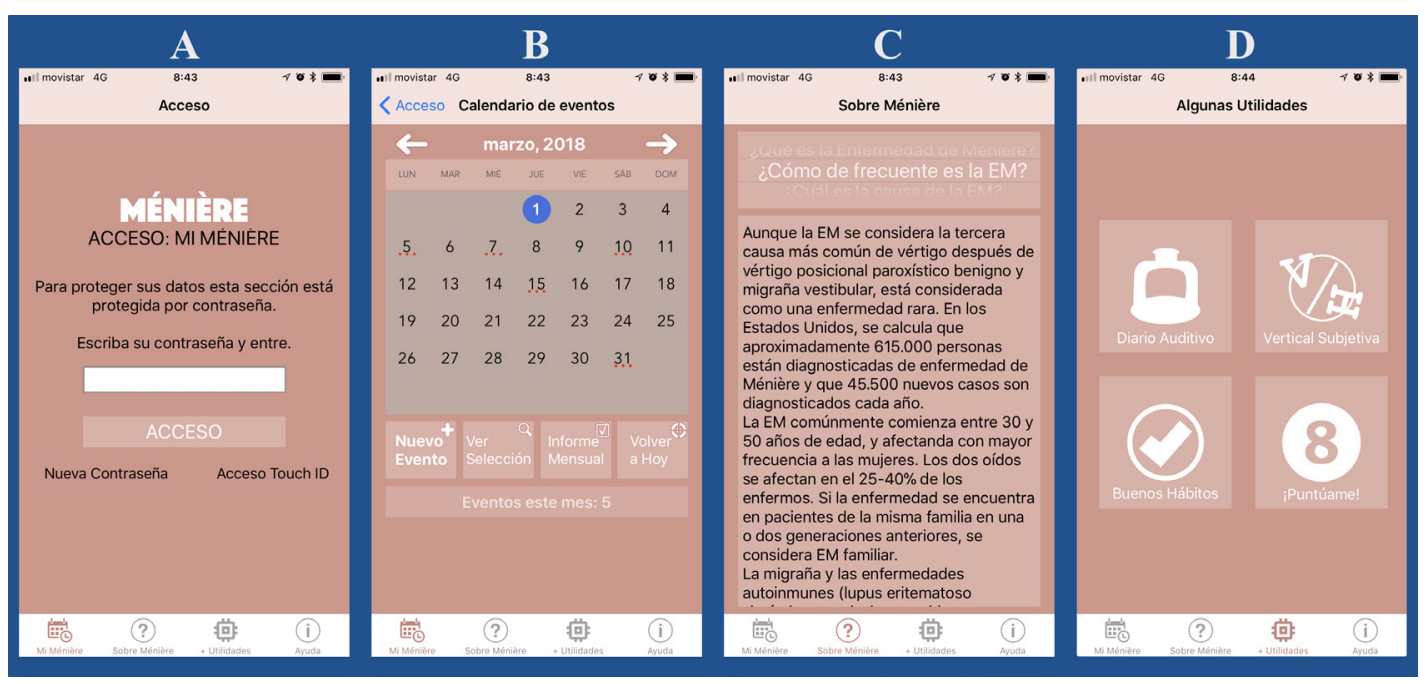

Figura 1. Pantallas principales de la aplicación Ménière. A: Pantalla de bienvenida protegida por contraseña alfanumérica o biométrica. B: Pantalla de eventos relacionados con la enfermedad de Ménière. C: Pantalla de información sobre la enfermedad de Ménière. D: Pantalla de utilidades para el control y seguimiento de la enfermedad de Ménière. Las pantallas mostradas han sido tomadas de la aplicación Ménière ejecutándose en un iPhone modelo 8.

- Pantalla de utilidades (Figura 1D): En esta pantalla se encuentran disponibles una serie de recursos informáticos que pueden ser de utilidad para el paciente con EM, parte de ellos han sido creados específicamente para esta aplicación como el diario auditivo y otros son recursos externos a la aplicación.

- Pantalla de ayuda y créditos: En esta sección se presenta la guía de uso de la aplicación y se incluye la información sobre de los diseñadores, los propietarios de la aplicación y las librerías de código informático externas utilizadas en la aplicación, estas bibliotecas se usaron en la creación del calendario y de los gráficos del informe mensual.

- De entre estas pantallas aquí resumidas detallaremos lo referente al diseño y funcionamiento de las pantallas de agenda de eventos y el diario auditivo, por ser las más específicamente relacionadas con el control de la EM.
Pantalla de eventos relacionados con la enfermedad de Ménière (Figuras 1B, 2A y 2B)

La acción principal que el usuario puede llevar a cabo en esta aplicación es el registro de eventos relacionados con la EM al cual se accede a través de la agenda incorporada en esta aplicación. Esta acción se permite realizar únicamente una vez al día y únicamente se puede añadir una acción en el día actual, no permitiéndose añadir acciones a otros días que no sean el actual. Con este método se pretendió que cada evento fuese un resumen de los hechos acontecidos a lo largo de ese día que el paciente relacione con su enfermedad, de modo análogo al proceso de escribir un diario en el cual al final del día se recogen en éste los eventos fundamentales del día.

La pantalla para añadir los efectos diarios de la enfermedad consta de dos secciones principales, una sección de síntomas agudos (Figura 2A) donde se recogen los principales síntomas relacionados con la crisis de EM, para muchos de ellos se 


\section{DISEÑO Y DESARROLLO DE UNA APLICACIÓN PARA DISPOSITIVOS MÓVILES PARA EL SEGUIMIENTO Y CONTROL DE LA ENFERMEDAD DE MÉNIẼRE REY-MARTÍNEZ J Y ESPINOSA-SÁNCHEZ JM}

establece el nivel de intensidad de estos a través de una sencilla escala analógica visual de intensidad. Hay dos síntomas agudos fundamentales siguiendo los criterios de la sociedad Bárány [4] para el diagnóstico de la enfermedad de Ménière que son el vértigo y los síntomas auditivos, así como una serie de síntomas asociados: inestabilidad, náuseas, dolor de cabeza, fotofobia, etc. Estos síntomas, además de valorarse en cuanto a su presencia e intensidad, son también estudiados en cuanto a los posibles desencadenantes ambientales que los han supuestamente producido o a los que el sujeto cree asociados. Se establecieron como desencadenantes el clima, el sueño, la actividad física, la alimentación, la cafeína, el alcohol y el tabaco.

Junto con estos síntomas agudos existen también disponibles para cada nuevo grupo de eventos un conjunto de «síntomas residuales» en el que se agrupan síntomas que el paciente puede experimentar no de manera aguda, sino de manera crónica o de forma persistente entre las crisis agudas. Estos síntomas son: mareo, inestabilidad, visión borrosa y presión cefálica. Para recoger la intensidad de estos síntomas crónicos se empleó una modificación de la escala de discapacidad para pacientes con alteraciones vestibulares desarrollada por Shepard [7]. Estos síntomas crónicos conforman la segunda sección de la pantalla añadir un nuevo episodio.

Además de poder añadir un evento cada día, el sujeto puede crear informes mensuales con los datos de los eventos acontecidos en cada mes. Estos informes resumen de manera gráfica y mediante análisis estadístico descriptivo y de agrupación los principales ítems presentes en cada evento (Figura 2B). Los informes generados para cada mes pueden ser enviados a voluntad del usuario siguiendo el método estándar para compartir archivos de imagen y texto presentes en el sistema operativo iOS.

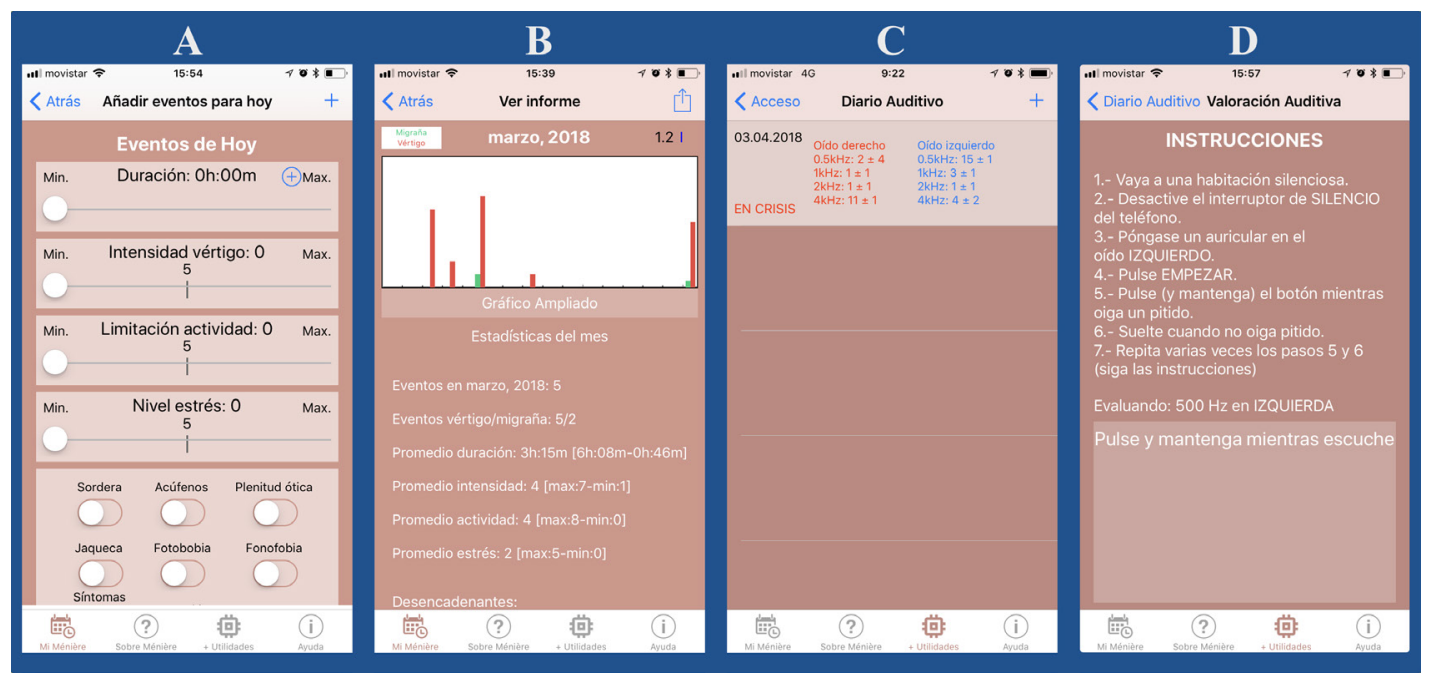

Figura 2. Pantallas de las herramientas de seguimiento. Se muestran en las pantallas A y B las pantallas correspondientes al seguimiento de las crisis y eventos relacionados con la enfermedad de Ménière. La pantalla A muestra la interfaz de introducción de las características agudas de las crisis para el día actual y la pantalla B muestra el informe gráfico y estadístico de las crisis que han aparecido durante un mes. La presencia de barras rojas significa días con crisis y la altura de la barra indica su intensidad general, las barras rojas muestran los días que ha habido síntomas migrañosos y su intensidad. Las pantallas $\mathrm{C}$ y D muestran el funcionamiento del diario auditivo. En la pantalla C se muestran los niveles auditivos obtenidos en los diferentes controles y en la pantalla $\mathrm{D}$ se muestra la interface de auto-prueba de audición basada en el método de von Békésy. Las pantallas mostradas han sido tomadas de la aplicación Ménière ejecutándose en un iPhone modelo 8. 


\section{DISEÑO Y DESARROLLO DE UNA APLICACIÓN PARA DISPOSITIVOS MÓVILES PARA EL SEGUIMIENTO Y CONTROL DE LA ENFERMEDAD DE MÉNIẼRE REY-MARTÍNEZ J Y ESPINOSA-SÁNCHEZ JM}

\section{Pantalla de diario auditivo (Figuras $2 \mathrm{C}$ y 2D)}

Para ayudar a llevar un control aproximado de los niveles auditivos del paciente se realizó un diseño de prueba auditiva, sin validez clínica contrastada, basada en el método de «autoaudiometría» sugerido por von Békésy [8]. En el método desarrollado, un sintetizador de tonos puros emite una señal sonora continua de nivel sonoro ascendente hasta que el sujeto empieza a oírla a través del auricular de inserción que se ha puesto en uno de sus oídos, en ese momento el sujeto presiona un botón que hace que el sintetizador empiece a descender el nivel sonoro de la señal (Figura 2D), cuando el sujeto deje de escuchar el sonido debe soltar el botón, momento en el cual el sintetizador comienza a subir de nuevo el nivel sonoro de la señal, esta operación se repetirá tres veces para cada frecuencia, las frecuencias de estímulo sonoro empleadas son: $500 \mathrm{~Hz}, 1000 \mathrm{~Hz}, 2000 \mathrm{~Hz}$ y 4000 $\mathrm{Hz}$. Estas frecuencias se miden para los dos oídos en cada sesión. Cuando la sesión de medición finaliza, la aplicación calcula el promedio de los tres intentos para cada frecuencia y muestra el resultado (Figura 2C).

Como sintetizador de sonido se emplearon exactamente los mismos ficheros con las clases escritas en Objective-C que controlan el sintetizador de sonido de la aplicación AudCal (Jorge Rey Martínez. España), que ha sido validada frente a los audiómetros de uso clínico mediante ensayo clínico controlado [9].

\section{Filosofía de diseño}

A la hora de diseñar una aplicación pensada para ser usada por pacientes en su día a día se establecieron una serie de criterios o principios de diseño sobre los que descansa toda la aplicación, estos son: privacidad, seguridad, usabilidad, veracidad y transparencia.

Ediciones Universidad de Salamanca / @D@®

\section{Privacidad y seguridad}

Si en general cualquier dato acerca del estado de salud de las personas ha de ser tratado con la máxima confidencialidad posible, a los diseñadores de esta aplicación les pareció especialmente importante la privacidad de los datos referentes a las crisis de vértigo que presentan los pacientes con enfermedad de Ménière. Así, aparte de los sistemas de control y privacidad que brinda el sistema operativo iOS por defecto, se añadieron una serie de medidas para incrementar los niveles de estos conceptos para esta aplicación:

- Se decidió no usar ningún servidor externo para preservar la información de cada usuario exclusivamente en su teléfono sin tener que recurrir a accesos y alojamientos en servidores de terceros que el usuario no controla, al menos al mismo nivel que su propio teléfono o tableta.

- Cada vez que la aplicación entra en segundo plano esta se cierra y al volver a activarse siempre lo hace en la pantalla de bienvenida.

- Tanto la pantalla de bienvenida como el acceso a la agenda y el acceso al diario auditivo están siempre, en cada nuevo acceso a la aplicación, protegidos por contraseña alfanumérica o biométrica.

- La base de datos está diseñada como un objeto del sistema de base de datos incluido por defecto en iOS (Core Data) participando así de las cualidades de protección y acceso que este sistema brinda, actualizándose además con futuras versiones del sistema operativo.

- Únicamente el usuario es quien decide y controla cuando, como y a quien se le envían sus informes mensuales.

\section{Usabilidad, veracidad y transparencia}

La aplicación cuenta con un diseño conforme a las líneas de usabilidad de aplicaciones recomendada por Apple para las aplicaciones de sus dispositivos (Human interface guidelines: https://developer. apple.com/design/). Además de estos criterios se 


\section{DISEÑO Y DESARROLLO DE UNA APLICACIÓN PARA DISPOSITIVOS MÓVILES PARA EL SEGUIMIENTO Y CONTROL DE LA ENFERMEDAD DE MÉNIÈRE REY-MARTÍNEZ J Y ESPINOSA-SÁNCHEZ JM}

contó para el diseño gráfico de la aplicación con una diseñadora profesional que se encargó de hacer una interface visualmente elegante a la vez que amistosa (Figuras 1 y 2 ).

De igual forma, para la versión en inglés se contó con un colaborador nativo de esta lengua que se encargó de revisar y corregir todos los textos de la aplicación para este idioma.

El contenido médico fue revisado por tres colaboradores médicos externos (especialistas en otorrinolaringología con especial dedicación y experiencia en el ámbito de la otoneurología). Los conceptos médicos incluidos en el diseño de la aplicación han tratado siempre de estar en consonancia con las publicaciones científicas y consensos actuales sobre la EM y sus circunstancias [3-9].

Igualmente, a lo largo del proceso de desarrollo de la aplicación se contó siempre con las impresiones de uso de varios pacientes diagnosticados de EM que nos permitieron comprobar y monitorizar si nuestro trabajo estaba cumpliendo con sus supuestas finalidades, esto nos permitió contar con una valoración de primera mano en cuanto a conocer lo más objetivamente posible el grado de sencillez y utilidad de nuestra aplicación por parte de los usuarios finales.

Como comentamos anteriormente, se limitó el número de eventos a uno al día, siendo además únicamente editable el día actual.

Toda la información acerca de los autores, colaboradores y principales referencias se encuentran incluidas en la pantalla de información de la propia aplicación.

\section{DISCUSIÓN}

La aplicación Ménière fue publicada en la App Store para dispositivos iOS a principios de 2017 tras haber conseguido implementar todas las funciones descritas en el apartado de material y métodos y pasado la revisión pertinente por parte de Apple previa a la publicación de cualquier aplicación en su App Store. Actualmente (marzo de 2018) se puede consultar y descargar la aplicación a través del enlace: https://itunes.apple.com/es/app/Ménière/ id1168430727? $\mathrm{mt}=8$. La adaptación en versión para Android está en fase de finalización.

La aplicación Ménière permite que el propio paciente pueda monitorizar la evolución de sus síntomas, haciéndose corresponsable de su salud. Igualmente, proporciona al médico especialista, a modo de calendario de vértigo, información detallada sobre la frecuencia, intensidad y duración de las crisis de vértigo junto con la presencia o no de otros síntomas acompañantes. Esta información es valiosísima, pues la EM exhibe un cuadro clínico bastante heterogéneo y no siempre es fácil el diagnóstico diferencial con otros trastornos que también ocasionan un síndrome vestibular episódico. Del mismo modo, posibilita evaluar la respuesta a un determinado tratamiento.

Como se describía en el apartado anterior, en el diseño de la aplicación se han primado una serie de cualidades en el diseño: privacidad, seguridad, usabilidad, veracidad y transparencia. Un diseño centrado en estas cualidades hace que la aplicación sea particular en cuanto a su uso y capacidades. El primer error de diseño que queríamos evitar era caer en una aplicación diseñada pensando en el profesional médico, aunque hemos intentado preservar la veracidad y transparencia que creemos que deben estar inequívocamente presentes en cualquier aplicación de salud o médica (aunque esta sea no profesional), no hemos querido hacer una aplicación compleja basada en conceptos médicos abstractos y con la jerga propia de la medicina, esto haría una aplicación muy técnica pero pensamos que también muy fría y distante para los pacientes que posiblemente terminarían no usando. Mantener este equilibrio de cercanía para el usuario y rigor médico no es sencillo y pensamos que para ello ha sido imprescindible contar con pacientes que a través de la Asociación Española de Enfermos con Enfermedad de Ménière (ASMES) voluntariamente han estado presentes a lo largo de todo el proceso de diseño y desarrollo haciéndolos llegar 


\section{DISEÑO Y DESARROLLO DE UNA APLICACIÓN PARA DISPOSITIVOS MÓVILES PARA EL SEGUIMIENTO Y CONTROL DE LA ENFERMEDAD DE MÉNIÈRE REY-MARTÍNEZ J Y ESPINOSA-SÁNCHEZ JM}

sus sugerencias y validando el uso amistoso de nuestra aplicación.

Otra peculiar característica presente en nuestra aplicación es la finalidad no investigadora ni económica que ésta tiene, el único beneficio que hay detrás de esta aplicación es de intentar contribuir a mejorar la calidad de vida de los pacientes. Si bien existen otras aplicaciones en el mercado actual, éstas han sido publicadas por instituciones privadas o por universidades con fines investigadores [7]. En nuestro caso quisimos que el paciente y su salud fueran los únicos beneficiarios de la aplicación.

Dentro de las anteriores cualidades en el proceso de diseño fue crítico de esta aplicación el establecer la base de datos físicamente en el propio teléfono o en hacerlo en un servidor interno, los diseñadores pensamos que difícilmente se puede lograr una aplicación controlada exclusivamente por los propios pacientes y alojarla en un servidor externo. Una base de datos local aporta una serie de limitaciones, como la difícil recuperación de los datos en caso de pérdida, por ejemplo, pero pensamos que es la mejor manera de conseguir una privacidad máxima para cada usuario.

En el desarrollo de la aplicación, hubo un punto que empeoraba ligeramente la experiencia de usuario, que fue la limitación de acceso por contraseña a la agenda de eventos y al diario auditivo. Como detallamos en material y métodos, este control se realiza no solo en el primer inicio de la aplicación, sino cada vez que ésta se recupera de un segundo plano, esta pequeña traba está en parte minimizada por el hecho de que el control de acceso basado en huella dactilar simplifica enormemente la tarea de tener que tener que teclear varias veces la contraseña según se use la aplicación.

Un aspecto innovador de esta aplicación es el control audiométrico incluido en ella. Cuando diseñamos la aplicación nos pareció un elemento llamativamente carente en otras aplicaciones, teniendo en cuenta que las aplicaciones auditivas han sido una de las primeras aplicaciones que aparecieron en la medicina aplicada a la $m$ Health, que quizás si esperamos que pueda arrojar algo de luz en futuras investigaciones sobre la EM.

Como ya hemos puesto de manifiesto en anteriores ocasiones [6], quizás el principal problema que nos ha traído toda la explosión de la medicina móvil es la falta de una legislación o entidades reguladoras que sirvan de guía y control de calidad entre la enorme cantidad de aplicaciones con fines supuestamente sanitarios que existen en la actualidad. La legislación y organismos controladores actuales como la Food and Drug Administration (FDA) americana, el marcado CE de la Comunidad Europea o la Agencia Española del Medicamento y Productos Sanitarios (AEMPS) han centrado sus esfuerzos en establecer los criterios y regulaciones aplicables a los productos sanitarios. Estrictamente hablando son los medios que a través de un soporte informático se emplea para diagnosticar, tratar o prevenir las enfermedades, centrándose en estas aplicaciones han dejado un vacío donde se encuentran una gran cantidad de aplicaciones para las que no existe ningún tipo de control por carecer de (o no ser tan evidente) este efecto diagnóstico, terapéutico o preventivo pero que sin embargo si son empleadas y demandados por la sociedad tecnológica actual.

\section{CONCLUSIONES}

Como principal limitación del presente trabajo resaltamos que éste es un artículo de carácter esencialmente teórico y descriptivo, pues no se ha realizado ningún control clínico o experimento material que confirme o desmienta las hipótesis y procesos aquí explicados. Es por tanto el único afán de este artículo el de trasladar al lector curioso los planteamientos y estrategias que se han desarrollado en el proceso de creación y publicación de una aplicación móvil dirigida a personas diagnosticadas de enfermedad de Ménière. Futuras investigaciones han de analizar la seguridad, privacidad, usabilidad de la aplicación y la satisfacción del paciente con ella. 


\section{DISEÑO Y DESARROLLO DE UNA APLICACIÓN PARA DISPOSITIVOS MÓVILES PARA EL SEGUIMIENTO Y CONTROL DE LA ENFERMEDAD DE MÉNIĒRE REY-MARTÍNEZ J Y ESPINOSA-SÁNCHEZ JM}

\section{AGRADECIMIENTOS}

Los autores de este artículo quieren muy sinceramente agradecer la desinteresada labor de las personas que han colaborado activamente en la creación de la aplicación Ménière: Mark Jackson, Marina González Llorca, Eva Perendreu, José Antonio López Escámez, Ángel Batuecas Caletrío, Nicolás Pérez Fernández y a la Asociación Síndrome de Ménière España (ASMES).

\section{DECLARACIÓN DE INTERESES}

Los autores de este artículo son los diseñadores, desarrolladores y propietarios de la aplicación Ménière. Los derechos (y posibles beneficios) de distribución y gestión de la aplicación han sido cedidos desinteresadamente por dichos autores en favor de la Asociación Síndrome de Ménière España (ASMES).

\section{BIBLIOGRAFÍA}

1. Sama PR, Eapen ZJ, Weinfurt KP, Shah BR, Schulman KA. An evaluation of mobile health application tools. JMIR Mhealth Uhealth. 2014;2:e19.

2. Becker S, Miron-Shatz T, Schumacher N, Krocza J, Diamantidis C, Albrecht UV. mHealth 2.0: experiences, possibilities, and perspectives. JMIR Mhealth Uhealth. 2014;2:e24.
3. Espinosa-Sánchez JM, López-Escámez JA. Ménière's disease. Handb Clin Neurol. 2016;137:257-77.

4. López-Escámez JA, Carey J, Chung WH, Goebel JA, Magnusson M, Mandalà M, et al. Diagnostic criteria for Menière's disease. J Vestib Res. 2015;25(1):1-7.

5. Frejo L, Soto-Varela A, Santos-Pérez S, Aran I, Batuecas-Caletrío A, Pérez-Guillen V, et al. Clinical Subgroups in Bilateral Ménière Disease. Front Neurol. 2016;24.7:182.

6. Frejo L, Martin-Sanz E, Teggi R, Trinidad G, SotoVarela A, Santos-Perez S, et al. Extended phenotype and clinical subgroups in unilateral Ménière disease: A cross-sectional study with cluster analysis. Clin Otolaryngol. 2017;42(6):1172-80.

7. Shepard NT, Telian SA, Smith-Wheelock M. Habituation and balance retraining therapy. A retrospective review. Neurol Clin. 1990;8(2):45975.

8. Békésy G. A New Audiometer. Acta Otolaryngol. 2009;35(5-6):411-22.

9. Larrosa F, Rama-López J, Benítez J, Morales JM, Martínez A, Alañón MA, et al. Development and evaluation of an audiology app for iPhone/iPad mobile devices. Acta Otolaryngol. 2015;135(11):1119-27.

10. Schmidt W, Sarran C, Ronan N, Barrett G, Whinney DJ, Fleming LE, et al. The Weather and Ménière's Disease: A Longitudinal Analysis in the UK. Otol Neurotol. 2017;38(2):225-33. 